\title{
EVALUACIÓN DE LAS CONSECUENCIAS DEL FUEGO EN QUEMAS CONTROLADAS MEDIANTE RADIOMETRÍA DE CAMPO
}

\author{
R. MONTORIO LLOVERÍA, F. PÉREZ CABELLO, \\ A. GARCÍA-MARTÍN y J. DE LA RIVA FERNÁNDEZ
}

Dpto. de Geografía y Ordenación del Territorio (Universidad de Zaragoza) Ciudad Universitaria, s.n. Zaragoza 50009

Correo electrónico de contacto: montorio@unizar.es

\begin{abstract}
RESUMEN. La alteración de las propiedades del suelo y la destrucción de la cubierta vegetal son dos importantes consecuencias inducidas por el fuego. A escala regional, los datos de teledetección son adecuados para hacer un seguimiento de los procesos posteriores al fuego. Sin embargo, a nivel de suelo, las investigaciones sobre cambios en los valores de reflectividad son necesarias para conocer las relaciones entre las consecuencias medioambientales generadas por el fuego y los valores de reflectividad espectral. Las medidas tomadas por medio de un radiómetro de campo proveen información útil para describir estas relaciones. El principal objetivo de este estudio es caracterizar los patrones temporales de los valores de reflectividad en un fuego experimental y analizar las relaciones entre estos valores y los cambios inducidos en el suelo. Para ello se llevó a cabo la quema controlada de dos parcelas experimentales registrando con una frecuencia mensual los valores de reflectividad por medio de un espectrómetro de campo. Los datos recogidos han mostrado cómo la cubierta vegetal experimenta su mayor cambio en el momento del fuego presentando una gran estabilidad en el periodo posterior en ausencia de regeneración vegetal, mientras que el suelo desnudo es la cubierta con alteraciones más acusadas en su comportamiento a partir de la situación postfuego debido al incremento de los procesos de erosión.
\end{abstract}

ABSTRACT. Alteration of soil properties and destruction of vegetation cover are two important fire-induced consequences. At regional scale, remote sensing data appears suitable for monitoring post-fire processes. However, at ground-level, researchs about changes on reflectance values are necessary in order to know relationships between fire-induced environmental consequences and spectral reflectance values. In this way, measurements taken by means of field spectroradiometer provide useful information for describing these relationships. The main objective of this study is to characterize temporal patterns of reflectance 
values in an experimental fire and to analyze relationships between spectral reflectance values and fire-induced soils changes. A prescribed fire was carried out in two experimental plots and reflectance measurements were taken with a monthly frecuency by using a field spectro-radiometer. The collected data have shown that vegetation cover undergoes its greater change in the moment of fire, showing a stable behaviour after the fire in absence of recovery processes. On the other hand, soil is the cover with most important alterations in its behaviour from the post-fire situation due to the increase of erosion processes.

Palabras clave: fuego experimental, efectos postfuego, espectrometría de campo, reflectividad.

Key words: experimental fire, post-fire effects, field spectrometry, spectral reflectance.

Enviado el 5 de mayo de 2006

Aceptado el 14 de septiembre de 2006

\section{Introducción}

El fuego es un factor ecológico de gran importancia en los ambientes mediterráneos. La respuesta de las áreas afectadas depende de la interacción de múltiples factores relacionados con las características físicas del medio afectado: tipo de vegetación, clima, topografía y características del suelo, y con las características del fuego en sí mismo: duración e intensidad, conceptos comúnmente empleados para evaluar los efectos del fuego.

La combustión de la cubierta vegetal y la alteración de las propiedades del suelo son dos de las consecuencias más importantes de los incendios forestales. La destrucción de la cubierta vegetal es quizás el más inmediato y observable, no obstante los mecanismos de rebrotamiento y semillado de la mayoría de las plantas mediterráneas conducen a un rápido proceso de cubrimiento vegetal, presentando características similares a las comunidades previas (Hanes, 1971; May, 1991).

Desde el punto de vista edafo-geomorfológico, el incremento de las tasas de erosión es una de las consecuencias más directamente ligadas al paso del fuego (Imenson et al., 1992; Pyne et al., 1996; Pardini et al., 2002; Cerdà y Lasanta, 2005). Este incremento es debido tanto a la desaparición del papel protector de la vegetación, con el consiguiente incremento de la energía cinética de la lluvia y su capacidad de destrucción, como a las elevadas temperaturas producidas durante el incendio que pueden alterar las propiedades químicas y físicas del suelo afectando su capacidad de infiltración y su resistencia a la erosión (Imenson et al., 1992; González-Pérez et al., 2004).

A escala regional la teledetección es una herramienta muy adecuada para estudiar los procesos postfuego debido a los cambios generados por el fuego en el comportamiento espectral del suelo y la vegetación (Patterson y Yool, 1998; Díaz-Delgado y Pons, 2001; Riaño et al., 2002). Índices espectrales tales como el Normalized Differenced Vegetation Index (NDVI) y el Normalized Burn Ratio (NBR) aplicados a imágenes de satélite han sido ampliamente 
empleados en el seguimiento de los cambios espaciales y temporales de la vegetación y en la estimación de características del fuego (Miller y Yool, 2002, Key y Benson, 2004).

Si bien es cierto que en los últimos años se han hecho importantes avances en la adquisición, procesamiento, análisis e interpretación de datos de alta resolución espectral procedentes de nuevos sensores hiperespectrales y de equipos de adquisición de datos hiperespectrales en el campo (Van Wagtendonk et al., 2004; Pérez-Cabello et al., 2005; Schaepman et al., 2005), la realización de estudios a escala de detalle no es tan abundante.

Las mediciones de reflectividad superficial en el campo pueden ser aplicadas en un gran número de aproximaciones analíticas de la teledetección como el análisis de mezclas espectrales (Smith et al., 2005), clasificación de imágenes de satélite, modelos predictivos, validación superficial (Zha et al., 2003), modelos de intercambio de energía (Chuvieco et al., 2005; Schaepman et al., 2005) o modelado de la reflectividad de la vegetación buscando longitudes de onda que incrementen la sensibilidad de los índices de vegetación o la creación de nuevos (Gitelson et al., 2002; Hansen y Schjoerring, 2003).

En este marco, la información recogida por un espectrómetro de campo sobre un fuego experimental resulta de gran utilidad para describir con precisión los efectos postfuego. Esta investigación tiene como objetivo caracterizar los patrones temporales de los valores de reflectividad en un fuego experimental y analizar las relaciones entre éstos y los cambios en el suelo y la vegetación generados por el fuego.

\section{2. Área de estudio}

La espectrometría de campo fue realizada en dos parcelas ubicadas en la estación experimental de Peñaflor (Zaragoza, España), construida en el marco del proyecto EROFUEGO (Fig. 1). Con unas dimensiones de 3x8 m se localizan en una ladera de orientación sur con pendiente de $12^{\circ}$. El clima puede calificarse de mediterráneo seco con una precipitación media anual de $300 \mathrm{~mm}$ y una temperatura media de $15^{\circ} \mathrm{C}$. El suelo es un regosol calcáreo desarrollado desde una terraza fluvial cuaternaria. La vegetación es un matorral mediterráneo dominado por semilladoras -Rosmarinus officinalis- y por herbáceas rebrotadoras -Brachypodium retusum-.

\section{Metodología}

\subsection{Quema controlada de las parcelas experimentales}

Las parcelas fueron sometidas el 16 de octubre de 2004 a las 12:00 de la mañana a un fuego controlado que se inició en la parte inferior de la parcela dejando que se propagara por su superficie de forma natural. De forma previa al incendio se colocaron junto a las parcelas dos grupos de termopares a diferentes profundidades $(6.5,2.5$ y $1 \mathrm{~cm})$ que permitieron un registro continuo de los cambios en la temperatura del suelo (Fig. 2). Asimismo, se contabi- 
lizó la duración total y la velocidad de propagación del incendio, estimando visualmente la altura de la llama. La intensidad del incendio se estimó a partir de las medidas de temperatura recogidas durante el mismo usando un termómetro sin contacto (MX 2 TD) (Fig. 2).

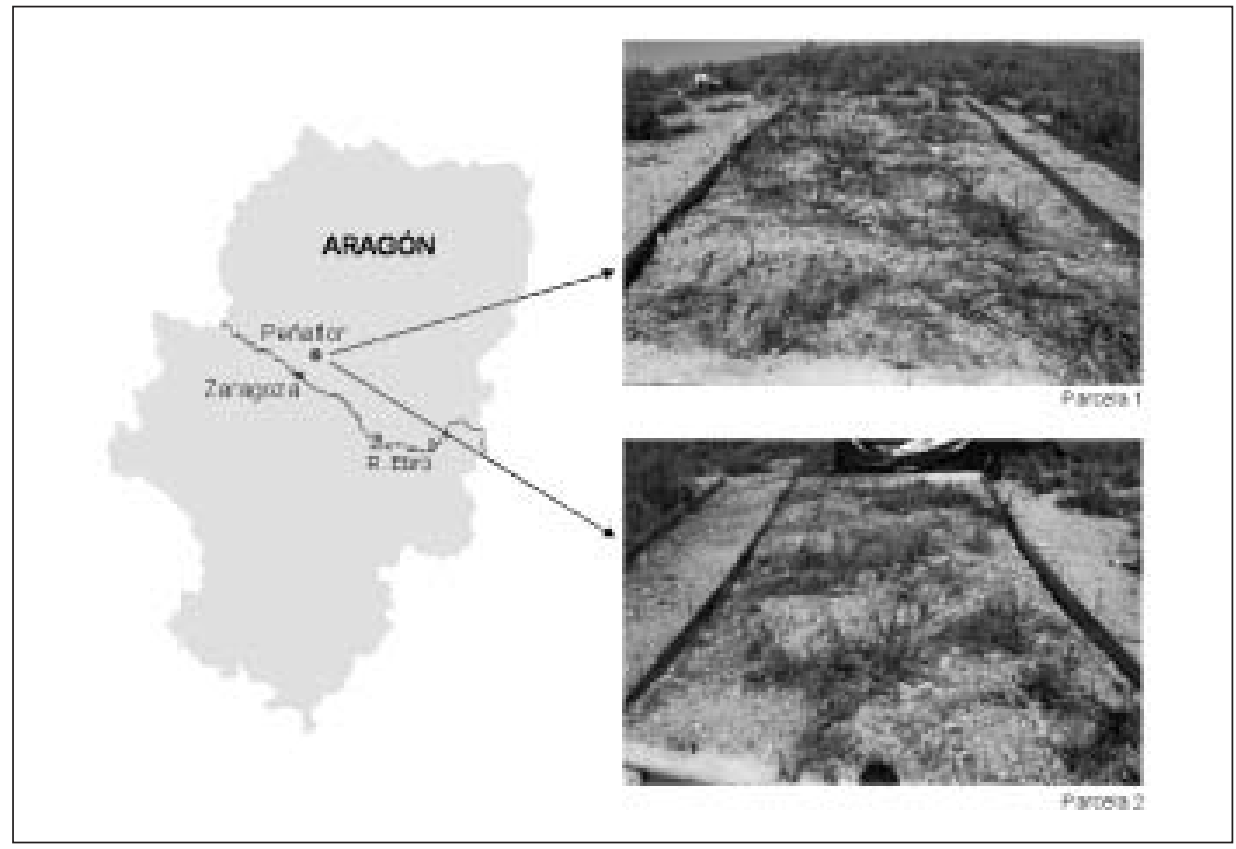

Figura 1. Área de estudio.
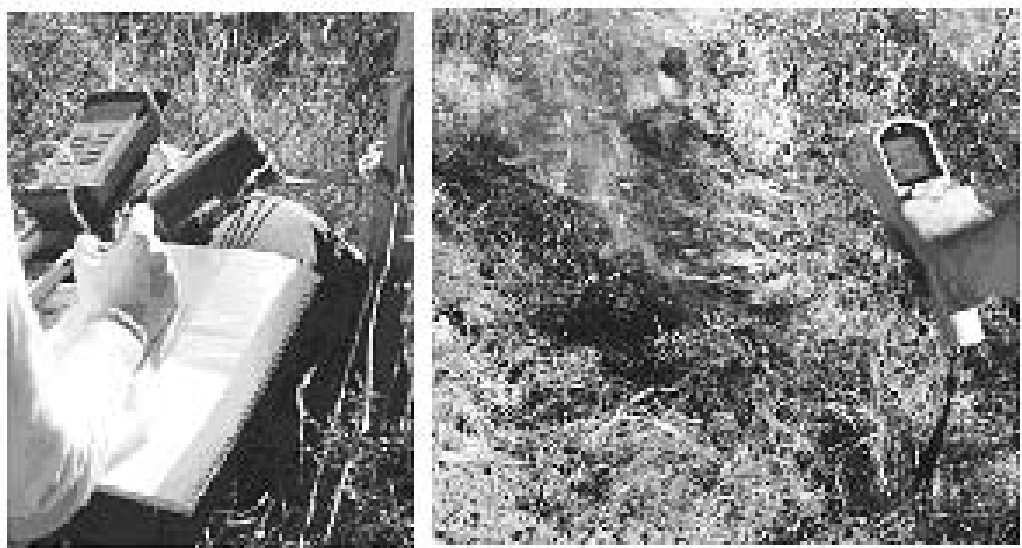

Figura 2. Registro de cambios en la temperatura del suelo por los termopares (izda.) y registro de temperaturas de superficie por el termómetro sin contacto (dcha.) 


\subsection{Medición de la reflectividad espectral}

La obtención de los datos de reflectividad se realizó sobre un conjunto de puntos distribuidos en las parcelas siguiendo un muestreo regular cada $80 \mathrm{~cm}$ en el eje vertical y $50 \mathrm{~cm}$ en el eje horizontal que generó un total de 109 puntos. La primera medición se realizó de forma previa al incendio. Inmediatamente después del mismo se tomó la medida correspondiente a la situación postfuego y, a partir de ese momento, durante un periodo de ocho meses se tomaron datos con una frecuencia mensual.

El equipo empleado está constituido por los siguientes elementos: (1) equipo portátil en el que se encuentra instalado el software OOIBase 32 compatible con el espectrómetro (Fig. 3a); (2) espectrómetro Ocean Optics USB2000-VIS-NIR con un rango útil de 400-900 nm y una resolución espectral de $0.5 \mathrm{~nm}$ (Fig. 3b); (3) fibra óptica de $2 \mathrm{~m}$ (Fig. 3c); (4) limitador de IFOV (Instantaneous Field of View) para fijar el campo de visión de la fibra óptica a un ángulo de $10^{\circ}$ (Fig. 3d) y (5) panel de referencia Spectralon de politetrafluoretileno (PTFE) con propiedades prácticamente lambertianas y altos valores de reflectividad (98\%) en un amplio rango de longitudes de onda (350-1800 nm) (Fig. 3e).

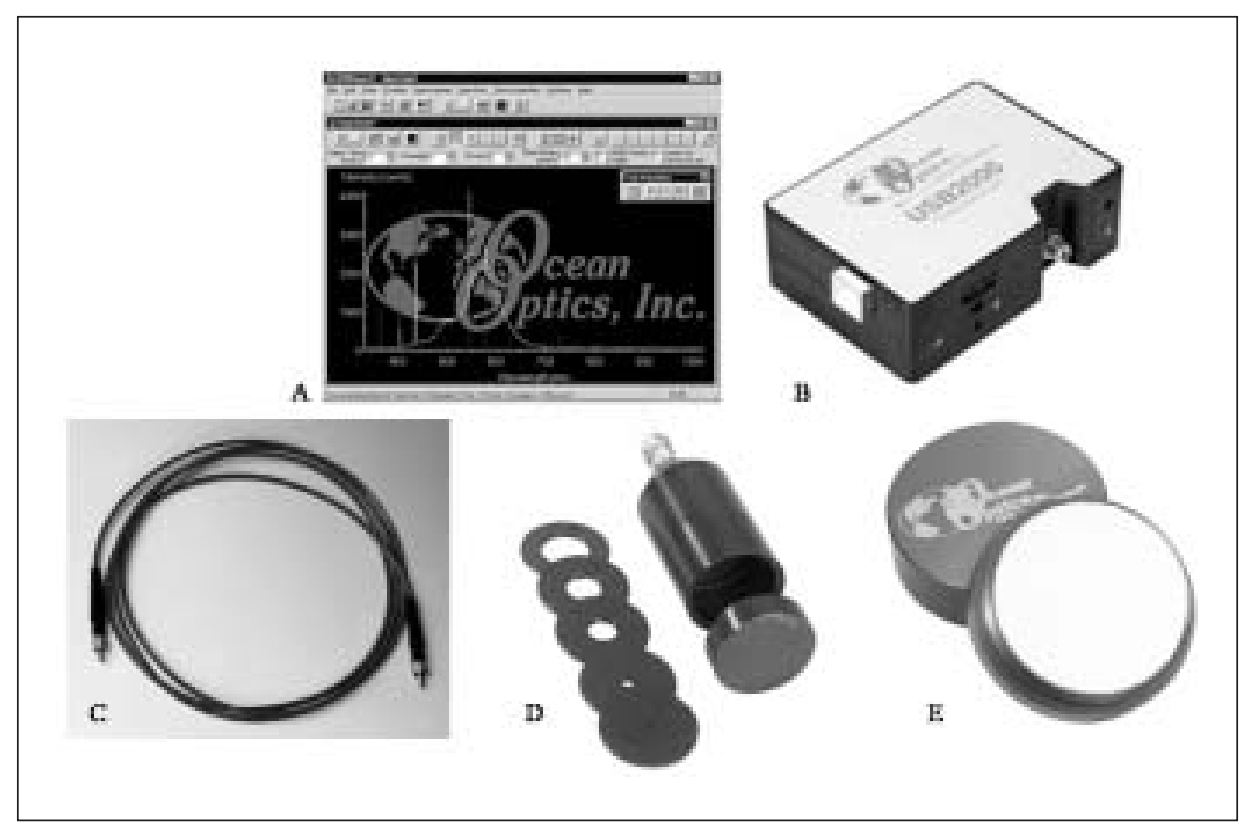

Figura 3. Instrumental para las mediciones de reflectividad

En la toma de mediciones de reflectividad se siguieron las recomendaciones básicas establecidas para la correcta adquisición de datos espectrales en el campo (Milton, 1987): (1) mantenimiento de la geometría entre sensor, panel de referencia y objetivo; 
(2) posicionamiento del sensor a 1,5 m sobre el nadir (altura dentro del intervalo recomendado que oscila entre 1-2 m); (3) homogeneidad en las mediciones: similares condiciones atmosféricas y buena iluminación, horario correspondiente al máximo solar (entre las 12 y las 14 h) para minimizar los efectos debidos a cambios en el ángulo solar y orden en la captura de información manteniendo la misma posición relativa respecto al sol y el resto de los elementos; y (4) comprobación previa de ausencia de interferencias exteriores como sombras sobre los puntos de muestreo o saturación por deslumbramiento de algún objeto exterior.

Además de estas recomendaciones la correcta realización de las medidas de reflectividad requiere el ajuste de algunos parámetros con influencia en la ratio señal-ruido, de forma que ésta tienda hacia valores 250:1. En este sentido, los siguientes ajustes fueron realizados en todas las jornadas de campo: (1) Medición con la fibra óptica no expuesta a la luz para registrar la señal correspondiente a la corriente oscura (dark current), señal eléctrica generada por los primeros 24 pixeles del espectrómetro y que no responde a la luz, para a continuación sustraer automáticamente ese espectro a las medidas posteriores; (2) Ajuste del tiempo de integración, que puede definirse como la frecuencia de conversión del espectrómetro o como el periodo de tiempo que el detector se encuentra expuesto a la luz entrante, buscando el valor más elevado posible para que la ratio señal-ruido sea elevada pero sin llegar a problemas de saturación (12-15 milisegundos en las medidas de este trabajo); (3) Promedio automático de espectros capturados por el sensor (20 mediciones promediadas en este trabajo).

Realizados estos ajustes, en las sucesivas jornadas de campo se procedió a capturar la información espectral de la superficie de $26 \mathrm{~cm}^{2}$ incluida en cada punto de muestreo (resultado de la combinación de la altura del sensor $-1,5 \mathrm{~m}-\mathrm{y}$ el ángulo de visión $-10^{\circ}$ ). La información se recogió en valores de reflectividad calculados por el espectrómetro mediante la siguiente fórmula:

$$
\% R_{\lambda}=\frac{S_{\lambda}-D_{\lambda}}{R_{\lambda}-D_{\lambda}} * 100
$$

donde $S_{1}$ es la radiancia del punto de muestreo en la longitud ${ }_{\lambda}, D_{\lambda}$ es la radiancia de la corriente oscura en la longitud $\lambda, \mathrm{y}_{\lambda}$ es la radiancia del panel de referencia en la longitud ${ }_{\lambda}$. Las óptimas y estables condiciones de iluminación y la breve duración de la captura de datos espectrales hicieron suficiente una única medida de $D_{\lambda} y R_{\lambda}$ en cada jornada de campo.

La información espectral obtenida en el campo fue posteriormente procesada en el laboratorio procediendo a su importación, filtrado y representación gráfica. El proceso de filtrado eliminó el ruido de las firmas espectrales manteniendo, al mismo tiempo, su variabilidad. Para ello se utilizó el filtro Savitzky Golay para firmas espectrales, un filtro unidimensional que aplica un ajuste de mínimos cuadrados a los datos recogidos en una ventana móvil que se desplaza a lo largo del espectro y cuyas dimensiones fueron especificadas para cada fecha en función del ruido visible en las curvas. 


\subsection{Estudio de la reflectividad espectral}

Dada la heterogeneidad superficial de las áreas capturadas en cada medición radiométrica, se seleccionaron aquellas mediciones que recogían de una manera más pura dos tipos de cubierta principales: suelo desnudo, 28 puntos, y vegetación, 45 puntos. A partir de los puntos homogéneos se seleccionó un punto representativo de cada cubierta para realizar en él el estudio temporal de los valores de reflectividad. Para ello se calculó la curva espectral media de la situación prefuego en cada categoría, se analizaron los residuales de cada punto respecto a la curva media y se seleccionó el punto con menor residual. El punto que fue seleccionado para la categoría vegetación recoge un área ocupada en el $90 \%$ de su superficie por cubierta vegetal activa, Rosmarinus officinalis denso con pequeños claros que dejan ver material fino del suelo. El área que corresponde al punto seleccionado de suelo desnudo se encuentra ubicada entre varias manchas de vegetación y está ocupada en igual proporción (50\%) por material fino y cantos rodados de diferente tamaño procedentes de material de terraza cuaternaria.

\section{Resultados}

\subsection{Características del fuego experimental}

La ausencia de continuidad del combustible hizo que la velocidad de propagación del fuego fuera muy lenta, aproximadamente de 20 minutos, alcanzando alturas de llama de 1,5 $\mathrm{m}$ aproximadamente y valores de transferencia de calor en el suelo en el rango $29-110^{\circ} \mathrm{C}$ según la información aportada por los termopares. Las temperaturas máximas registradas durante el incendio se encuentran mayoritariamente en el rango $400-800^{\circ} \mathrm{C}$, mostrando la segunda parcela un mayor número de puntos en los rangos de intensidad superiores a $600^{\circ} \mathrm{C}$.

\subsection{Cambios en el comportamiento espectral de las cubiertas}

\subsubsection{Vegetación}

La situación prefuego de esta cubierta es la de una vegetación activa (Fig. 4, izda.) y así lo refleja su comportamiento espectral con valores de reflectividad que muestran el contraste característico de la vegetación entre el VIS y el NIR -red edge-. La curva presenta bajos valores en el espectro visible -VIS-, mostrando un ligero incremento en la longitud de onda del verde respecto al azul y el rojo por la acción de los pigmentos foliares, y valores elevados en el infrarrojo próximo -NIR- indicando una adecuada estructura foliar (Fig. 5).

El fuego provoca la total desaparición de la vegetación quedando la superficie de estos puntos cubierta por cenizas negras o blancas en función de la menor o mayor severidad del incendio (Fig. 4, centro). Este cambio se manifiesta en los valores de reflecti- 
vidad con una total homogeneización de la curva, que adopta un perfil prácticamente horizontal propio de la cubierta de cenizas, con valores más o menos elevados en función de la mayor o menor presencia de cenizas blancas (Fig. 5).

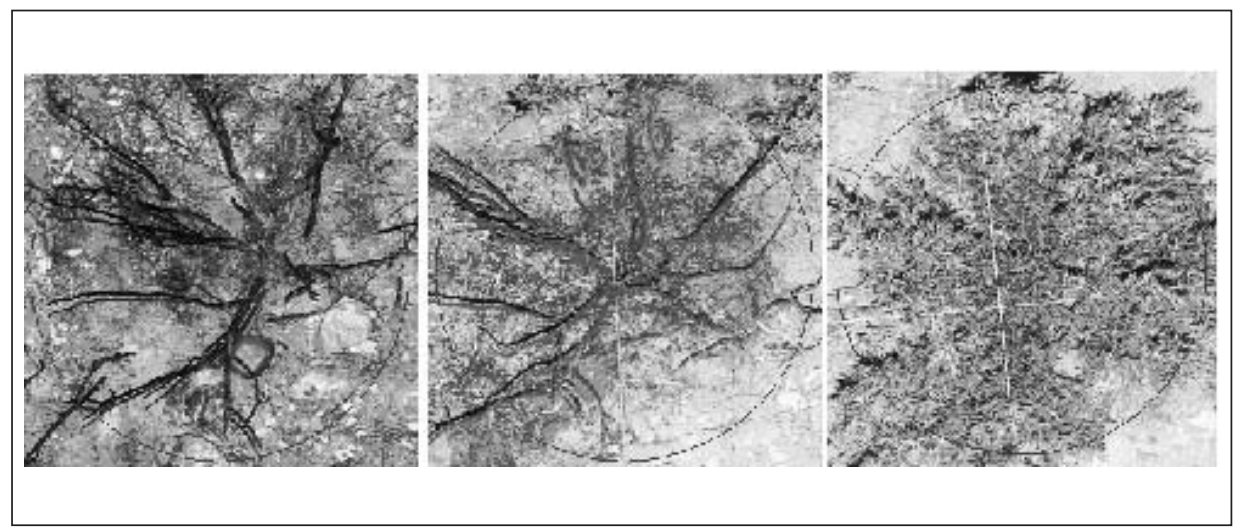

Figura 4. Evolución visual del punto seleccionado de vegetación (prefuego, postfuego y 2 meses)

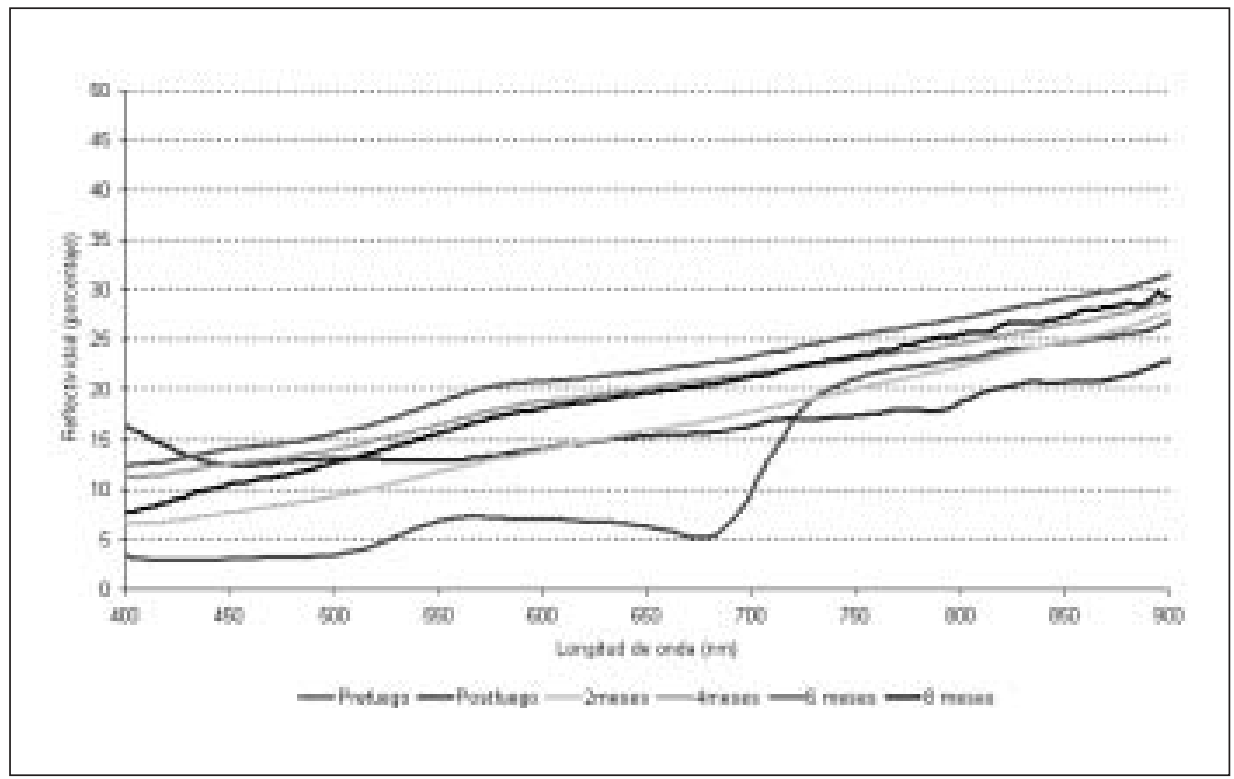

Figura 5. Evolución de la reflectividad del punto de vegetación 
A partir del momento postfuego, el cambio más importante observable en estos puntos es la eliminación progresiva de la capa de cenizas por efecto de la acción de la lluvia, la escorrentía superficial y el viento, dejando al descubierto el suelo subyacente (Figura 4, dcha.). Así pues, la curva a partir de los dos meses adopta el perfil de suelo desnudo, con valores de reflectividad que se incrementan de forma progresiva con la longitud de onda (Figura 5). Este perfil lo mantiene durante el resto de la serie temporal indicando la ausencia de procesos de regeneración vegetal hasta el momento temporal estudiado en este trabajo. Las variaciones en el periodo 2-8 meses se producen en la misma proporción en todas las longitudes de onda produciéndose desplazamientos de la curva en el eje vertical. Estas variaciones, con cambios nunca superiores al 5\%, obedecen a las variaciones en el contenido de humedad del suelo. Los valores más elevados se registran en el intervalo correspondiente a un período de fuerte sequía en la zona de estudio y las medidas con valores más bajos corresponden en el tiempo con fenómenos de lluvia registrados por los pluviómetros de la estación experimental.

\subsubsection{Suelo desnudo}

La situación prefuego de esta cubierta es la de un suelo desnudo con presencia de material fino y escasa pedregosidad superficial (Fig. 6, izda.). Esta cubierta muestra en este momento temporal la curva espectral característica con cierta pendiente debido al incremento de los valores de reflectividad conforme incrementa la longitud de onda (Fig. 7).

En el momento del incendio esta cubierta no experimenta cambios muy profundos al no verse afectada directamente por él (Fig. 6, centro). La posible presencia de cenizas procedentes de los puntos próximos de vegetación quemada y el incremento de temperatura originado por el fuego explican el cambio observado en la firma espectral, con menor pendiente y mayores valores de reflectividad (Fig. 7).

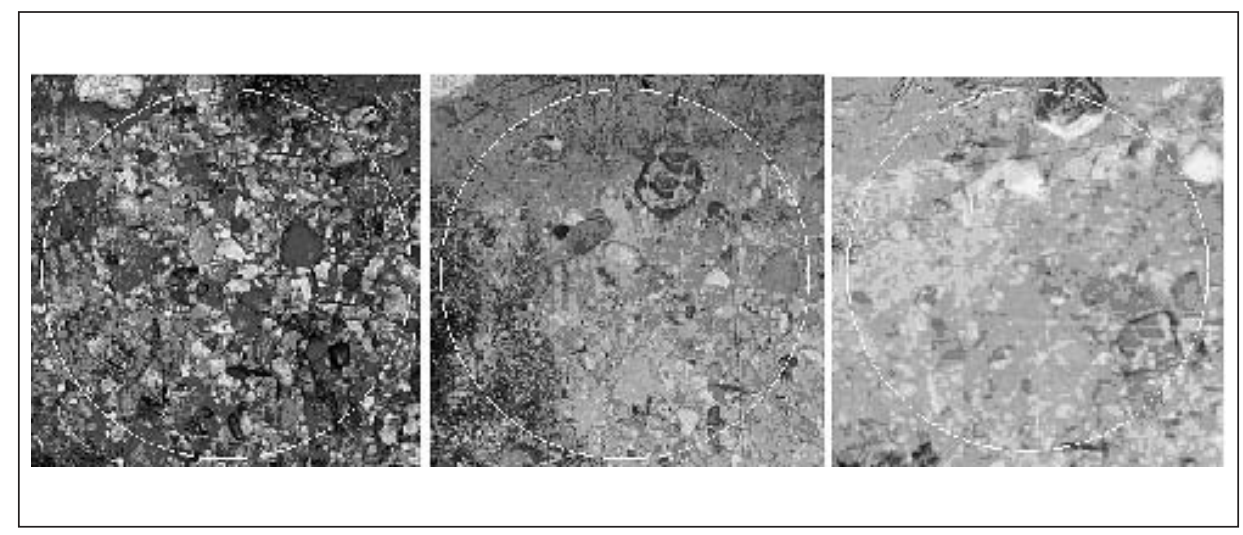

Figura 6. Evolución visual del punto de suelo desnudo (prefuego, postfuego y 2 meses) 


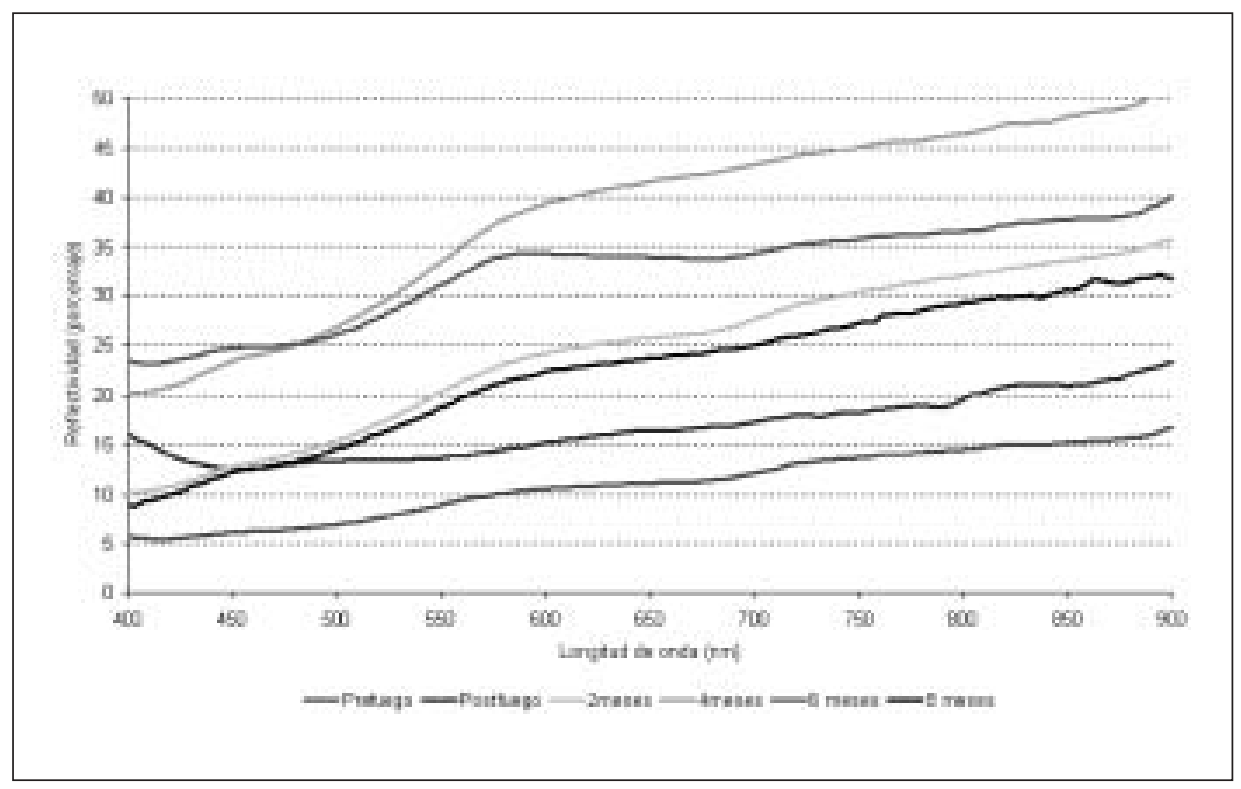

Figura 7. Evolución de la reflectividad del punto de suelo desnudo

Los cambios más importantes en el suelo desnudo se producen asociados a los procesos postfuego. La desaparición de la cubierta vegetal en zonas próximas genera un incremento de los procesos erosivos que eliminan el material fino del suelo dejando al descubierto el material grueso subyacente e incrementando la pedregosidad superficial (Fig. 6, dcha.). Estos importantes cambios hacen que la cubierta suelo desnudo experimente grandes contrastes en sus valores de reflectividad a partir del momento temporal postfuego (Fig. 7). En las medidas posteriores al fuego la firma espectral de estos puntos incrementa su pendiente y sus valores de reflectividad. Las variaciones en estos valores se explican, al igual que en los puntos de vegetación, por variaciones en el contenido de humedad del suelo, con valores más elevados en los momentos de sequía (4 y 6 meses) e inferiores en momentos de presencia de humedad en el suelo ( 2 y 8 meses).

\subsection{Comparación temporal de los dos tipos de cubierta}

Este análisis comparativo entre los valores de reflectividad de los dos tipos de puntos en un momento temporal concreto permite conocer los cambios relativos entre ellos (Fig. 8).

La situación previa al fuego refleja un claro contraste entre los puntos de vegetación y los puntos de suelo desnudo, mostrando ambas categorías sus firmas espectrales características. La situación postfuego muestra el efecto homogeneizador del fuego al igualarse el comportamiento espectral de ambas cubiertas. El suelo desnudo, en este momento, sólo se ha visto afectado por el fuego de forma indirecta por la presencia de cenizas proceden- 


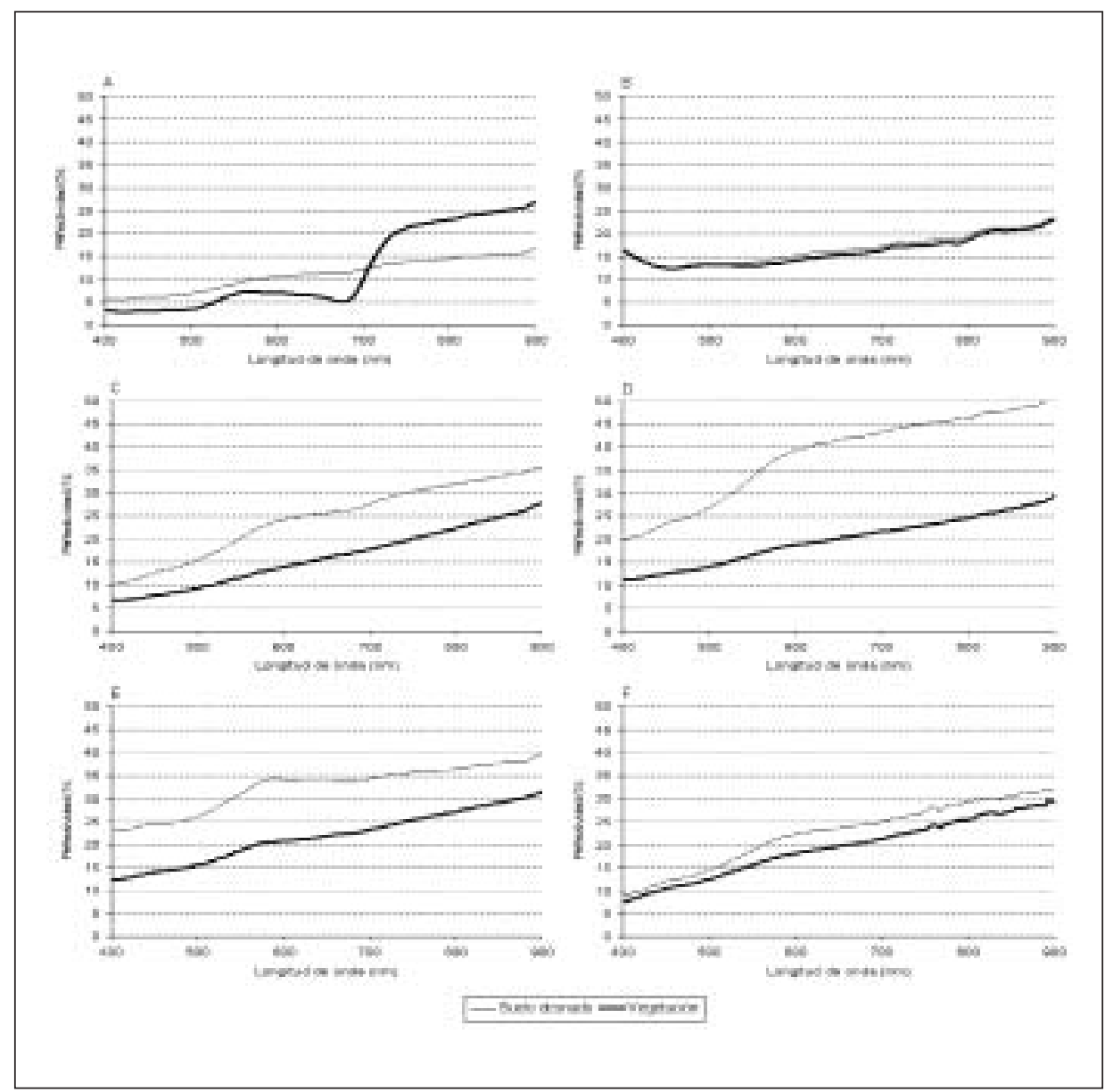

Figura 8. Comparación de los valores de reflectividad de los dos puntos representativos en diferentes momentos temporales: A) Prefuego, B) Postfuego, C) 2 meses,

D) 4 meses, E) 6 meses, F) 8 meses

tes de la combustión de la vegetación, de ahí que los cambios en su comportamiento espectral sean leves. Sin embargo, la vegetación experimenta en este momento postfuego el cambio más profundo adoptando un comportamiento espectral propio de las cenizas.

A partir de este momento las firmas espectrales de las dos cubiertas se mantienen estables en su morfología produciéndose únicamente cambios en sus valores de reflectividad traducidos en desplazamientos proporcionales en el eje vertical. El incremento y descenso en los valores de reflectividad se produce de forma solidaria en los dos tipos de puntos respondiendo, como se ha comentado anteriormente, al contenido de humedad en el suelo. El punto de vegetación experimenta cambios leves con modificaciones en la reflectividad que no superan diferencias superiores al 5\% entre fechas. Su comporta- 
miento a partir del momento postfuego puede considerarse, por lo tanto, como estable, hasta que se produzca el proceso de regeneración vegetal, momento en el que la firma espectral de estos puntos irá adoptando progresivamente la que mostraban en la situación prefuego. El suelo desnudo presenta, sin embargo, unos cambios mucho más acusados con modificaciones que alcanzan el $15 \%$. Estos cambios lo sitúan con valores de reflectividad muy superiores a los puntos de vegetación desde el momento temporal postfuego hasta la medida correspondiente a los 8 meses, donde vuelve a registrar valores de reflectividad similares a los puntos de vegetación. Esta cubierta es pues la que experimenta mayores alteraciones a partir del momento del postfuego, ya que al desaparecer la acción protectora de la vegetación se ve muy afectada por los procesos erosivos que suponen la desaparición de la fracción fina del suelo y el incremento de la pedregosidad superficial, manifestándose estos cambios en los valores de reflectividad reflejados por esta cubierta.

\section{Conclusiones}

Durante los ocho meses siguientes a la realización del fuego experimental se han observado importantes cambios en las cubiertas presentes en las parcelas experimentales, cambios que tienen su reflejo en el comportamiento espectral de estas cubiertas. La información obtenida de los gráficos de reflectividad, con el apoyo visual de fotografías, permite concluir que las diferencias espectrales existentes entre las dos cubiertas de ocupación del suelo previas al fuego desaparecen como consecuencia de éste. Se uniformiza el comportamiento espectral de la parcela con firmas propias del suelo desnudo. A pesar de esta homogeneidad en las firmas espectrales se observa un comportamiento muy diferenciado a partir del momento postfuego entre los puntos que previamente eran suelo desnudo y los que eran vegetación. En la cubierta vegetal, destruida en su práctica totalidad por el incendio y convertida en suelo desnudo, no se observan cambios importantes más allá de la medida de postfuego debido a la ausencia de procesos de regeneración vegetal. Sin embargo, los cambios observados en el suelo desnudo son especialmente importantes en los meses posteriores al incendio debido a la reactivación y aparición de nuevas microrredes de escorrentía superficial capaces de eliminar la fracción fina del suelo incrementando la pedregosidad superficial. Este último extremo pone de manifiesto la conectividad de los parches de vegetación y áreas de suelo desnudo en zonas semiáridas.

El análisis realizado ha permitido observar la influencia del contenido de humedad en las variaciones en los niveles de reflectividad. Las mediciones realizadas en periodos temporales de ausencia de humedad presentan valores superiores que los correspondientes a periodos con presencia de humedad en el suelo. La sensibilidad a las condiciones de humedad edáfica es mucho mayor en las zonas de suelo desnudo original que en los puntos de suelo desnudo resultado de la eliminación de la vegetación. Este hecho puede explicarse por la presencia en estos puntos de restos de necromasa vegetal que matiza el comportamiento del suelo desnudo.

Desde el punto de vista metodológico los resultados de este trabajo muestran la utilidad de la información procedente de la radiometría de campo para el estudio de los cambios generados por el fuego en las diferentes cubiertas y el estudio del proceso de regeneración vegetal. 


\section{Agradecimientos}

Este estudio fue financiado con una beca del Gobierno de Aragón con el soporte del Ministerio de Ciencia y Tecnología español a través del proyecto RS-FIRE (CGL200504863) PIR-FIRE (PIP098/2005). Este proyecto tiene como objetivo el análisis de los procesos de erosión y regeneración vegetal post-incendio con distintos métodos y a dos escalas de trabajo: escala de detalle con parcelas y escala regional mediante imágenes de satélite Landsat TM/ETM+. En este trabajo se muestran los resultados correspondientes a una de las líneas metodológicas seguidas en este proyecto, la referente al estudio mediante radiometría de campo a escala de detalle. Los resultados aquí mostrados corresponden a un periodo temporal que no alcanza el momento final de la investigación por lo que las conclusiones presentadas deben contemplarse desde esta perspectiva.

\section{Referencias bibliográficas}

CERDÀ, A. y LASANTA, T. (2005). Long-term erosional responses after fire in the Central Spanish Pyrenees: Water and sediment yield. Catena, 60: 59-80.

Chuvieco, E., Riaño, D., Danson, F.M., Bowvier, P. y Martin, P. (2005). Use of canopy reflectance models to simulate burn severity levels. En Proceedings of the 5th International Workshop on remote sensing and GIS applications to forest fire management: fire effects assessment. Zaragoza, 233-238.

DíAZ-Delgado, R. y Pons, X. (2001). Spatial patterns of forest fires in Catalonia (NE of Spain) along the period 1975-1995. Analysis of vegetation recovery after fire. Forest Ecology and Management, 147: 67-74.

Gitelson, A., Kaufman, Y., Stark, R. y Rundquist, D. (2002). Novel algorithms for remote sensing estimation of vegetation fraction. Remote Sensing of Environment, 80: 76-87.

GonzÁlez-PÉrez, J.A., GonZÁlez-Vila, F.J., Almendros, G. y Knicker, H. (2004). The effect of fire on soil organic matter - a review. Environment International, 30: 855-870.

HANES, T.J. (1971). Succession after fire in the chaparral of southern California. Ecol. Monogr., 41: 27-52.

HANSEN, P. y SCHJOERRING, J. (2003). Reflectance measurement of canopy biomass and nitrogen status in wheat crops using normalized difference vegetation indices and partial least squares regression. Remote Sensing of Environment, 86: 542-553.

Imeson, A.C., Verstraten, J.M., VAn Mulligen, E.J. y SeVinK, J. (1992). The effects of fire and water repellency on infiltration and runoff under Mediterranean burnt forest (Spain). Catena, 19: 345-362. 
Key, C.H. y Benson, N.C. (2004). Landscape Assessment (LA) Sampling and Analysis Methods. [http://www.fire.org./firemon/lc.html]

MAY, T. (1991). Observaciones y reflexiones sobre el comportamiento tras fuego de algunas especies de la zona mediterráneas de Andalucía Oriental. Ecología, 5: 125-134.

Milton, E. (1987). Principles of field spectroscopy. International Journal of Remote Sensing, 8(12): 1807-1827

MilleR, J.D. y Yool, S.R. (2002). Mapping forest post-fire canopy consumption in several overstory types using multi-temporal Landsat TM and ETM+ data. Remote Sensing of Environment, 82: 481-496.

PARDINI, G., GiSPERT, M. y DUNJO, G. (2002). Relative influence of wildfire on soil properties and erosion processes in different Mediterranean environments in NE Spain. Science of the Total Environment, 328: 237-246.

PATTERSON, M.W. y YoOL, S.R. (1998). Mapping fire-induced vegetation mortality using Landsat Thematic Mapper data: a comparison of linear transformation techniques. Remote Sensing of Environment, 65: 132-142.

Pérez-Cabello, F., Montorio, R., García-Martín, A. y De la Riva, J. (2005). Methodology approach for analyzing fire-induced soils and vegetation changes using a spectral analysis system and high spatial resolution photograph. En Proceedings of the 5th International Workshop on remote sensing and GIS applications to forest fire management: fire effects assessment. Zaragoza, 263-267.

Pyne, S.J., ANDREWS, P.L. y LAVEn, R.D. (1996). Introduction to Wildland Fire. John Wiley and Sons, New York.

Riaño, D., Chuvieco, E., Ustin, S., Zomer, R., Dennison, P., Roberts, D. y Salas, J. (2002). Assessment of vegetation regeneration after fire through multitemporal analysis of AVIRIS images in the Santa Monica mountains. Remote Sensing of Environment, 79: 60-71.

Schaepman, M., Koetz, B., Schaepman-Strub, G. y Itten, K. (2005). Spectrodirectional remote sensing for the improved estimation of biophysical and chemical variables: two case studies. International Journal of Applied Earth Observation and Geoinformation, 6: 271-282.

Smith, A., Wooster, M., Drake, N., Dipotso, F., FAlKowski, M. y HudAK, A. (2005). Testing the potential of multi-spectral remote sensing for retrospectively estimating fire severity in African Savannahs. Remote Sensing of Environment, in press.

VAN WAGTENDONK, J., Rooy, R. y KeY, C.H. (2004). Comparison of AVIRIS and Landsat ETM+ capabilities for burn severity. Remote Sensing of Environment, 92: 397-408.

ZHA, Y., GAO, J., NI, S., LIU, Y., JiANG, J. y WEI, Y. (2003). A spectral reflectance-based approach to quantification of grassland cover from Landsat TM imagery. Remote Sensing of Environment, 87: 371-375. 